\title{
Factors affecting the haptic filled-space illusion for dynamic touch
}

\author{
Abram F. J. Sanders • Astrid M. L. Kappers
}

Received: 29 July 2008 / Accepted: 24 October 2008 / Published online: 12 November 2008

(C) The Author(s) 2008. This article is published with open access at Springerlink.com

\begin{abstract}
In the haptic filled-space illusion for active dynamic touch, observers move their fingertip across an unfilled extent or an extent filled with intermediate stimulations. Previous researchers have reported lengths of filled extents to be overestimated, but the parameters affecting the strength of the illusion are still largely unknown. In the current research, we show that the illusion persists when intermediate stimulations do not provide information about the extent's length. In addition, the results show that the strength of the illusion increases with the number of filler elements. In contrast with earlier research, we control for movement speed differences between filled and unfilled extents. The results suggest that the strength of the illusion is independent of the overall average movement speed. Insight into factors affecting the strength of the illusion may provide a better understanding of the kinematic mechanisms underlying haptic length perception.
\end{abstract}

Keywords Illusions $\cdot$ Tactile $\cdot$ Touch $\cdot$ Psychophysics

\section{Introduction}

In the long-known visual filled-space illusion, observers judge a linear extent that is filled with discrete filler elements to be longer than an unfilled extent of the same length (also known as Oppel-Kundt illusion; e.g., Coren and Girgus 1978). Although less studied, various haptic analogs of the filled-space illusion have been identified

A. F. J. Sanders $(\bowtie) \cdot$ A. M. L. Kappers

Physics of Man, Helmholtz Institute, Utrecht University, P.O. Box 80 000, 3508 TA Utrecht, The Netherlands

e-mail: a.f.j.sanders@uu.nl over the years (Craig 1931; Dresslar 1893; Jaensch 1906; Parrish 1893; Révész 1934; Suzuki and Arashida 1992). Filled extents in the haptic case include punctured cardboard cards, raised-line gratings, wooden laths with holes cut out on the edges, and so on.

One might find the coexistence of illusory effects of filled extents in both the visual and haptic modality to be striking; this suggests, for example, that the haptic illusion might be mediated through the visualization of what is being touched and would thus be essentially visual in nature. However, geometric illusions have been reported for congenitally blind people who have had no visual experience (e.g., Heller et al. 2002). Moreover, a number of researchers have argued that close ties exist between the way a haptic stimulus is explored by the hand and the way it is perceived (e.g., Davidson 1976; Gibson 1962; for an overview, see Appelle 1991). In the case of the haptic filled-space illusion, filled extents are overestimated when participants actively move their finger across the stimuli (active dynamic touch), whereas filled extents are underestimated when stimuli are impressed onto the skin of the forearm (static touch; Craig 1931; Parrish 1893). This raises the question regarding the nature of intermediate stimulations in the haptic filled-space illusion.

In this study, we investigate the haptic filled-space illusion for active dynamic touch. Previously, Révész (1934) mentioned the existence of the illusion but did not provide quantitative data. Suzuki and Arashida (1992) measured Points of Subjective Equality (PSE) in a discrimination experiment, but used a reference length of only a single wavelength. Likewise, Jaensch (1906) tested filled extents of a single wavelength in a reproduction experiment. Only the study by Dresslar (1893) provides indications concerning parameterization of the illusion. He used punctured cardboard cards, with the results suggesting that 
overestimations increase with the number of punctures. In all of these studies, the movement speed of the tracing finger was not controlled for.

Increasing evidence shows that haptic length perception is based on observing kinematic variables. For example, observers who were instructed to trace linear extents at high speeds judged these lengths to be shorter than the same extents traced at considerably lower speeds (Hollins and Goble 1988; Von Skramlik 1933; Wapner et al. 1967). In the case of free exploration, linear extents oriented radially from the body were judged to be longer than extents oriented tangentially; at the same time, it was observed that the extents that were judged longer were traced at slightly lower speeds, which led to a corresponding increase in movement time (Armstrong and Marks 1999; Wong 1977). Similarly, convex lengths were overestimated compared to concave lengths; again, these overestimations correlated with differences in movement time that were, in turn, due to slight speed differences between the various surface types (Sanders and Kappers 2008). Thus, one explanation for the haptic filled-space illusion is that filled extents are traced at slightly lower speeds.

However, findings of Dresslar (1893) suggest otherwise. He succeeded in conducting his experiment under conditions of passive dynamic touch (cardboard being moved underneath stationary fingertip), thus being able to make movement times exactly the same. Interestingly, he reported the illusion to persist. However, one should be cautious in comparing active and passive touch conditions.

A confounding factor in all of these studies is that the end points of extents are the same as the filler elements. Thus, for unfilled extents, the puncture the finger comes across after having passed over the first one is the puncture marking the end point. On the other hand, for filled extents, the observer has to move the index finger one wavelength beyond the last puncture to be sure that the extent has ended. Consequently, this might lead the participant to overestimate filled extents. If so, doubts are cast on whether the illusion is actually one of overestimation of filled space. Interestingly, none of the studies has noted this possible end point effect, while biases reported are of the same order of magnitude (Suzuki and Arashida 1992).

In this research, we investigated the filled-space illusion in a more thorough and systematic way than has previously been done. In Experiment 1, we investigated whether the overestimations of filled extents compared with unfilled extents increase with the number of filler elements. Filled extents are defined to be extents with discrete intermediate stimulations at the fingertip tracing the stimuli. We also investigated whether overall movement speed has an effect on the strength of the illusion. In Experiment 2, participants directly compared filled extents of different filler densities. None of the studies mentioned have tested direct compari- sons of filled extents. If we find that overestimations in Experiment 1 increase with the number of filler elements, we expect filled extents to be overestimated compared to less densely filled extents in the case of direct comparisons in Experiment 2. In both experiments, we excluded end point effects by using easily identifiable end points that are clearly distinguishable from filler elements. In addition, movement speeds within a trial have been made the same through the use of a metronome.

\section{Materials and methods}

\section{Participants}

The participants consisted of five males and two females who were in their 20 s or early 30 s, all of whom were righthanded. They participated in both Experiment 1 and Experiment 2. Participants were naive as to the aims and design of the experiments, and were paid for their efforts.

\section{Stimuli and setup}

Stimuli were linear extents of varying lengths defined by two small rubber pads glued onto sheets of swell paper (Fig. 1a). The rubber pads were spherical caps with a diameter of $8 \mathrm{~mm}$ and a height of approximately $2 \mathrm{~mm}$. Between the two rubber pads, we printed raised spatial patterns (width $2 \mathrm{~cm}$ ). These patterns were either gratings with wavelengths $(\lambda)$ of 4 or $8 \mathrm{~mm}$, or raised blocks. In Experiment 1, participants discriminated lengths of raised blocks from lengths of gratings of either of the two wavelengths; in Experiment 2, they discriminated lengths of 4-mm gratings from lengths of 8-mm gratings. Gratings consisted of raised lines with a width of $1 \mathrm{~mm}$. When moving the finger across the stimuli, end points were readily identifiable. Nine comparison lengths $(L)$ were available: $8.8,9.6,10.4,11.2,12,12.8,13.6,14.4$, and $15.2 \mathrm{~cm}$. Note that comparison lengths are multiples of the two wavelengths used, so that the distribution of raised lines is uniform over the entire stimulus.

Upon stimulus presentation, swell paper sheets were placed into a stimulus holder that was fixed onto a tabletop. Participants were comfortably seated behind the table, their right shoulder being approximately on the setup's midline. On either side of the stimulus holder, two movement stops were attached (small wooden blocks). The distance between the two movement stops was $28 \mathrm{~cm}$. Stimuli were touched with the fingertip of the right index finger. Observers were instructed to make smooth movements between the movement stops, touching the stimulus on the way. A metronome was ticking at a frequency of 1 or $2 \mathrm{~Hz}$. To induce observers to move with equal speeds across the different stimuli, they were required to synchronize each 

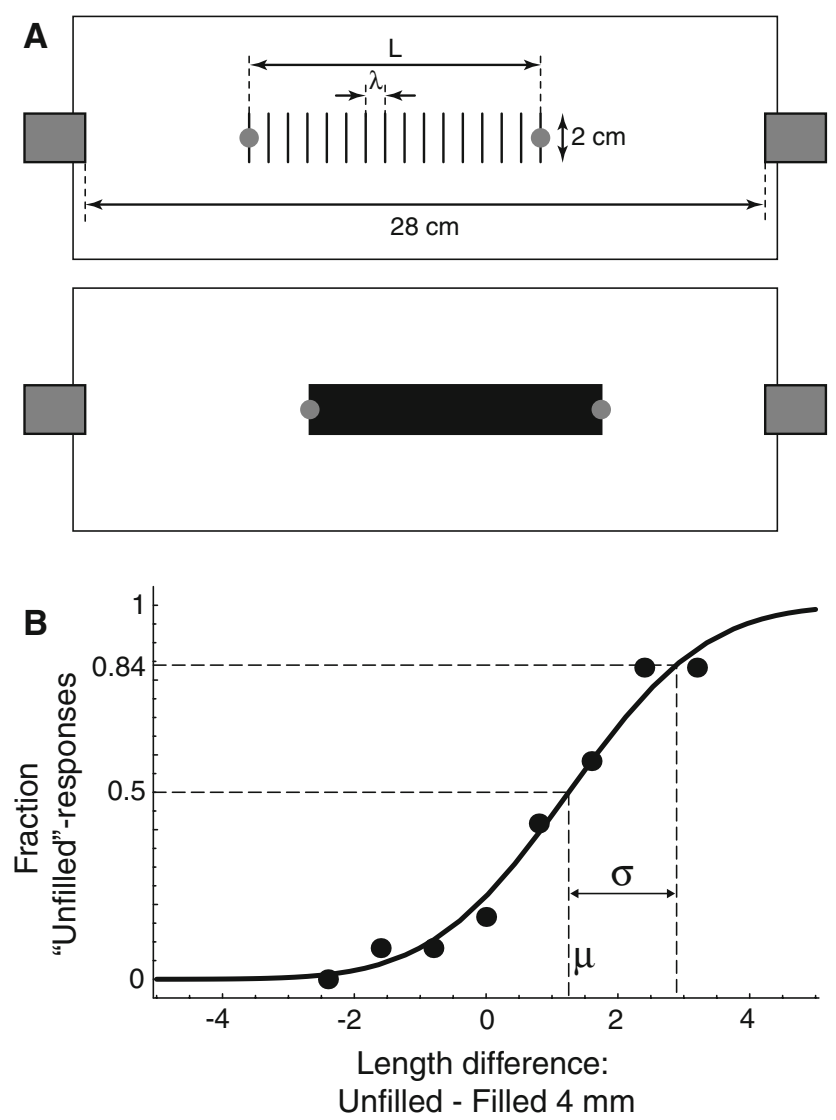

Fig. 1 a Stimuli. Participants moved their index finger between two stops, pacing movement speed through the use of a metronome. End points of filled and unfilled extents were rubber pads that were easily distinguishable from raised filler patterns. Stimulus lengths were randomly displaced to the left or right of the center of the setup by a constant amount. b Psychometric curve. A typical example of a psychometric curve obtained in this study is shown. Parameter $\mu$ represents the Point of Subjective Equality

tick of the metronome with arrival of the index finger at the left or right movement stop.

The midpoint of the stimulus was randomly located $1.25 \mathrm{~cm}$ to the left or right of the setup's midpoint to prevent observers from using the locations of the end points as length cues. Talcum powder was used to reduce friction between the finger and the rubber pads. The participants wore soundproof headphones that blocked auditory cues from the finger moving across the grating, while at the same time permitting the ticks of the metronome to pass. Participants were also blindfolded. Halfway through the experiment, we replaced the stimulus set to prevent wearout of the raised-line drawings.

Design and procedure

A trial proceeded as follows. Participants put their right index finger against the left movement stop on the first stimulus sheet and traced the stimulus a number of times, keeping pace with the ticks of the metronome. Participants lifted their hands while the experimenter quickly changed the swell paper sheets, and the participants explored the second stimulus. They then decided which of the two stimuli felt longer. Generally, participants traced a stimulus back and forth three or four times.

We measured psychometric functions according to the two-alternative forced-choice (2AFC) method using constant stimuli. The reference length was $12 \mathrm{~cm}$ throughout. The length difference between the two types of stimuli that were tested in the discrimination experiment served as the independent variable. In Experiment 1, participants compared raised blocks (U unfilled) with gratings ( $F$ filled), where the length difference is defined as U-F. In Experiment 2, participants compared gratings of wavelengths 4 and $8 \mathrm{~mm}$; the length difference is defined as F8-F4. For example, a length difference of $+2.4 \mathrm{~cm}$ in Experiment 1 corresponded to the following four combinations: U-14.4 cm, F-12 cm; F-12 cm, $\mathrm{U}-14.4 \mathrm{~cm} ; \mathrm{U}-12 \mathrm{~cm}, \mathrm{~F}-9.6 \mathrm{~cm}$; and F- $9.6 \mathrm{~cm}, \mathrm{U}-12 \mathrm{~cm}$. The two lengths of a stimulus pair were presented in both time orders to avoid response biases, and the reference length was a raised block or a grating to prevent any learning effects. Based on pilot experiments, we anticipated positive biases in all conditions. Therefore, we added a length difference of $+3.2 \mathrm{~cm}$ to the distribution of sample points to make it appear more symmetric to the observer (Fig. 1b).

The set containing the four possible combinations for each of the eight possible length differences was presented three times, amounting to a total of 96 trials per psychometric curve. Trials were independently randomized within each of the three blocks. A psychometric curve was measured in a single session lasting about $1.5 \mathrm{~h}$; sessions were done on separate days. Before commencing the actual experiment, participants were given a practice session to get accustomed to the setup and use of the metronome. Every session started with a few practice trials. Participants were never given any feedback.

In Experiment 1, participants compared raised blocks with gratings of different wavelengths. This experiment consisted of four conditions that were formed by the combination of the two factors of wavelength $(4 \mathrm{~mm} / 8 \mathrm{~mm})$ and metronome frequency $(1 \mathrm{~Hz} / 2 \mathrm{~Hz})$. In Experiment 2, participants discriminated lengths of gratings with wavelengths of $8 \mathrm{~mm}$ from wavelengths of $4 \mathrm{~mm}$. Two conditions were tested, which corresponded to the factor of metronome frequency $(1 \mathrm{~Hz} / 2 \mathrm{~Hz})$. To prevent learning effects, all six conditions of Experiments 1 and 2 were mixed and randomized across observers.

\section{Data analysis}

Cumulative Gaussians were used as psychometric functions. The two parameters $\mu$ and $\sigma$ (Fig. 1b) are interpreted 
as the PSE and the discrimination threshold, respectively. All $p$ values reported are two-tailed, unless explicitly stated otherwise.

\section{Results}

\section{Experiment 1}

Figure 2a shows average PSEs for the four conditions in Experiment 1 . Biases were significantly different from zero in all cases, with $t_{6}=3.18, p=0.009 ; t_{6}=3.61, p=0.006$; $t_{6}=3.14, p=0.010$; and $t_{6}=2.79, p=0.016$ for conditions U-F4/1 Hz, U-F4/2 Hz, U-F8/1 Hz, and U-F8/2 Hz, respectively (one-tailed $p$ values). Positive biases indicate that the unfilled extent had to be longer than the grating in order for the two to be perceived as equally long. Thus, observers overestimated lengths of gratings. A two-by-two ANOVA (wavelength $\times$ movement speed) with a repeated-measures design showed a significant main effect of wavelength $\left(F_{1,6}=6.95, p=0.039\right)$ but no main effect of movement speed $\left(F_{1,6}=0.79, p=0.409\right)$. There was no significant interaction effect $\left(F_{1,6}=0.11, p=0.752\right)$. Average biases for conditions U-F4 and U-F8 were $1.08 \mathrm{~cm}$ and $0.85 \mathrm{~cm}$, respectively. This corresponds to an overall average effect size of $8.4 \%$.

There were no effects on the thresholds of wavelength or movement speed. A repeated-measures analysis showed that the main effects of wavelength and movement speed and their interaction were non-significant $\left(F_{1,6}=2.05\right.$, $p=0.202 ; F_{1,6}=2.78, p=0.146$; and $F_{1,6}=0.44, p=0.531$, respectively). The overall average threshold was $2.13 \mathrm{~cm}$.

\section{Experiment 2}

Figure $2 b$ shows average PSEs for the two conditions in Experiment 2. Biases were significantly different from zero in both cases, with $t_{6}=4.06, p=0.003$ and $t_{6}=3.40$, $p=0.007$ for conditions 1 and $2 \mathrm{~Hz}$, respectively (onetailed $p$ values). Positive biases indicate that a grating with a wavelength of $8 \mathrm{~mm}$ had to be longer than a grating with a wavelength of $4 \mathrm{~mm}$ in order for the two to be perceived as equally long. Lengths of 4-mm gratings are thus overestimated compared to $8-\mathrm{mm}$ gratings. A pairedsamples $t$ test showed that there was no significant effect of movement speed $\left(t_{6}=0.10, p=0.928\right)$. The average bias in Experiment 2 was $0.88 \mathrm{~cm}$, which corresponds to an effect size of $7.6 \%$.

As in Experiment 1, there was no effect of movement speed on thresholds, as shown by a paired-samples $t$ test $\left(t_{6}=1.02, p=0.349\right)$. The average threshold in Experiment 2 was $1.77 \mathrm{~cm}$. A paired-samples $t$ test on average thresholds per observer in Experiments 1 and 2 showed that
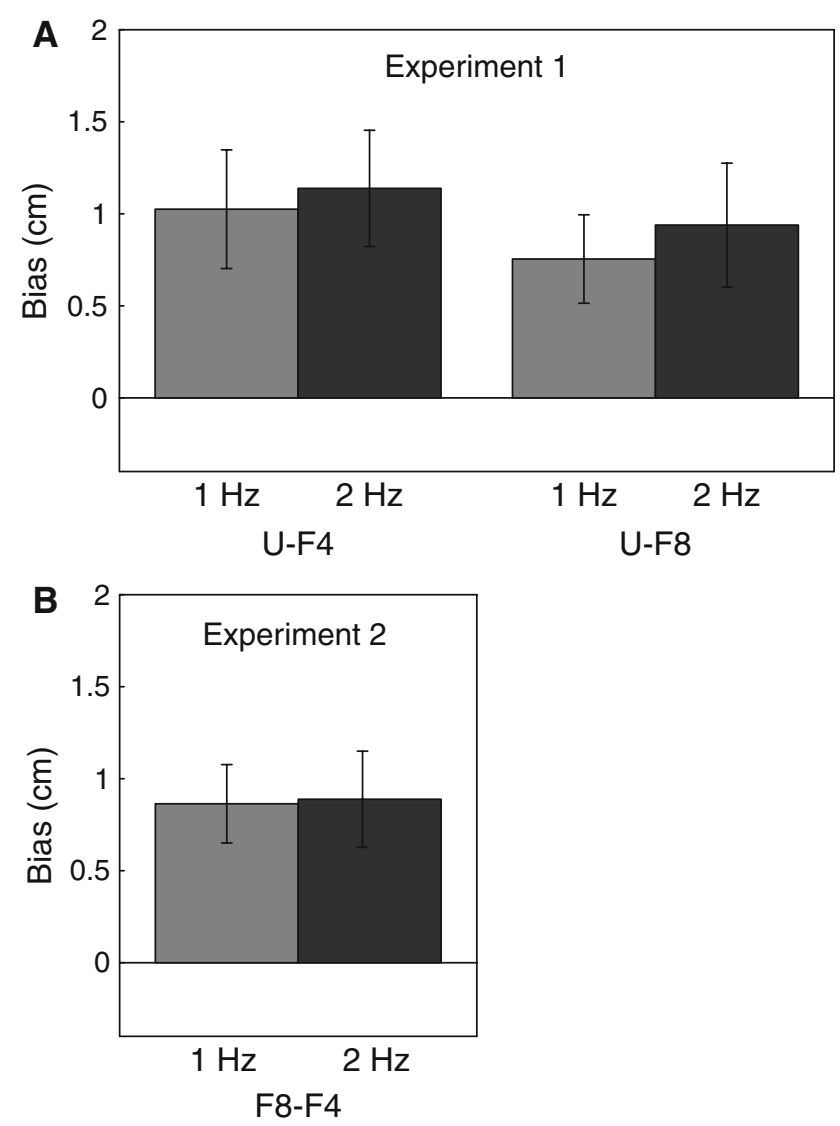

Fig. 2 Average Points of Subjective Equality. Error bars indicate standard errors of the mean. a Conditions in Experiment 1: UnfilledFilled $4 \mathrm{~mm}$ and Unfilled-Filled $8 \mathrm{~mm}$ at metronome frequencies of 1 and $2 \mathrm{~Hz}$. b Conditions in Experiment 2: Filled $8 \mathrm{~mm}-$ Filled $4 \mathrm{~mm}$ at metronome frequencies of 1 and $2 \mathrm{~Hz}$

thresholds in Experiment 2 were significantly smaller $\left(t_{6}=3.93, p=0.008\right)$.

\section{Discussion}

The results show that the lengths of extents filled with discrete filler elements are overestimated compared to less densely filled or unfilled extents. The strength of the illusion depends on the filler density. The amount of overestimation increases with the number of filler elements, as shown by the main effect of wavelength in Experiment 1 and the significant biases in Experiment 2. The results corroborate findings by Dresslar (1893). Importantly, these overestimations cannot be due to end point effects, and one might therefore interpret the illusion as a spatial distortion. Furthermore, we controlled for movement speed, so that speeds were the same for the two types of stimuli within a trial. As discussed in the Introduction, kinematic properties of hand movements play a determining role in haptic length perception. However, we found no evidence for an effect of 
doubling movement speed on the strength of the illusion in either experiment.

Although the intermediate stimulations at the fingertip are essentially non-informative about the length of the extent (the two end points suffice), we find that they affect perceived length. Moreover, the effects of filler density show that the intermediate stimulations affect perceived length parametrically, which raises the question as to the basis for the haptic filled-space illusion. Since the physical movement speed for the two stimuli within a trial was equal, the question is whether increased stimulation at the fingertip directly distorts the perception of kinematic variables of speed or time.

Since movement duration is a primary cue for perceived length, one could argue that filler elements affect perceived time. A number of studies have reported a filled-duration illusion, i.e., filled time intervals judged longer than empty ones (Buffardi 1971; Craig 1973; Goldstone and Goldfarb 1963; Grimm 1934; Otto Roelofs and Zeeman 1951; Thomas and Brown 1974). The illusion has been shown for the auditory modality (clicks and continuous tones), visual modality (flashes and continuous light), and tactile modality (vibrations applied to the index finger; short bursts or continuous vibrations). Thomas and Brown (1974) used different frequencies for end point tones and filler tones, and Buffardi (1971) showed that overestimations increase with the number of filler elements in all three modalities. Thus, if the haptic filled-space illusion for dynamic touch is essentially a variant of the filled-duration illusion, the influence of filler density in the former case must have a temporal character.

On the other hand, studies on tactile roughness perception show that discrimination performance is remarkably independent of scanning speed (Bensmaïa and Hollins 2003; Lamb 1983; Lederman 1974; Lederman 1983; Meftah et al. 2000; Smith et al. 2002; Vega-Bermudez et al. 1991). Thus, textures are generally perceived as equally rough, no matter how fast observers move their hands over the texture. This holds for both active and passive dynamic touch. More closely related to the present study are the experiments on frequency discrimination of gratings by Nefs et al. (2002), who remarked "there was considerable variation [in scanning speed] between and within trials" (p. 979). The studies on roughness perception and frequency discrimination of gratings suggest a different mechanism. The filler elements may affect perceived spatial extent, or the length signal after movement speed has been accounted for.

Another explanation for the haptic filled-space illusion could be that movement speed is perceived differently for different gratings. Two subsystems contribute to the sensation of speed of limb movement: proprioceptive stimulation in muscles and joints, and cutaneous stimulation at the contact between finger and stimulus. Interestingly, a recent study by Dépeault et al. (2008) investigated the ability to scale slip velocities through the use of a cylindrical drum rotating underneath the stationary fingertip. The surface of the drum could be smooth or covered with raised-dot patterns. For regular textures, the surface having a spatial period of $8 \mathrm{~mm}$ was perceived as moving slower than surfaces having a smaller spatial period $(2-3 \mathrm{~mm})$. In addition, smooth surfaces were perceived as moving slower than the textured surfaces. These effects are in agreement with the findings of the present paper. For example, consider a 4- and 8-mm grating of the same length. We used a metronome to make actual movement speeds constant across all stimulus types. Movement duration for these two stimuli is then the same, but perceived speed for the latter grating is lower (perception of duration is veridical by assumption). The length of the 4-mm grating would thus be overestimated, which is exactly what we found. However, the question is whether the effects on slip speed reported by Dépeault et al. (2008) are large enough to account for the present findings.

We found no effect of wavelength and movement speed on thresholds. Discrimination thresholds are a measure of the precision or reliability of observers' judgments. Apparently, the rubber pads that indicated the end points of the stimulus lengths were equally well discernable in all conditions. The finding of significantly lower thresholds in Experiment 2 is surprising, as this effect cannot be due to learning effects. Apparently, the precision with which grated lengths can be discriminated from each other is higher than when a grated length is compared with a raised block.

In conclusion, this study provides one of the first systematic investigations of parameters affecting the haptic filledspace illusion for dynamic touch. In contrast with previous studies, we have controlled for end point effects and withintrial differences in movement speed. We have shown that non-informative intermediate stimulations at the fingertip affect perceived length. The strength of the effect increases with the amount of intermediate stimulation. Since we know from previous research that length perception is primarily based on a kinematic mechanism, the increased stimulations possibly distort perception of kinematic variables of speed and time. Thus, the haptic filled-space illusion offers another opportunity to study the general principles underlying length estimation through dynamic touch.

Acknowledgments This research was supported by the Netherlands Organization for Scientific Research (NWO).

Open Access This article is distributed under the terms of the Creative Commons Attribution Noncommercial License which permits any noncommercial use, distribution, and reproduction in any medium, provided the original author(s) and source are credited. 


\section{References}

Appelle S (1991) Haptic perception of form: activity and stimulus attributes. In: Heller MA, Schiff W (eds) The psychology of touch. Lawrence Erlbaum Associates, New Jersey, pp 169-188

Armstrong L, Marks LE (1999) Haptic perception of linear extent. Percept Psychophys 61:1211-1226

Bensmaïa SJ, Hollins M (2003) The vibrations of texture. Somatosens Mot Res 20:33-43

Buffardi L (1971) Factors affecting the filled-duration illusion in the auditory, tactual, and visual modalities. Percept Psychophys 10:292-294

Coren S, Girgus JS (1978) Seeing is deceiving: the psychology of visual illusions. Lawrence Erlbaum Associates, New Jersey

Craig FE (1931) IV. Variations in the illusion of filled and unfilled tactual space. Am J Psychol 18:112-114

Craig JC (1973) A constant error in the perception of brief temporal intervals. Percept Psychophys 13:99-104

Davidson PW (1976) Haptic perception. J Pediatr Psychol 1:21-25

Dépeault A, Meftah E-M, Chapman CE (2008) Tactile speed scaling: contributions of time and space. J Neurophysiol 99:1422-1434

Dresslar FB (1893) III. Experiments on open and filled space for touch. Am J Psychol 6:332-342

Gibson JJ (1962) Observations on active touch. Psychol Rev 69:477-491

Goldstone S, Goldfarb JL (1963) Judgment of filled and unfilled durations: intersensory factors. Percept Mot Skills 17:763-774

Grimm K (1934) Der Einfluß der Zeitform auf die Wahrnehmung der Zeitdauer. Z Psychol 132:104-132

Heller MA, Brackett DD, Wilson K, Yoneyama K, Boyer A, Steffen H (2002) The haptic Müller-Lyer illusion in sighted and blind people. Perception 31:1263-1274

Hollins M, Goble AK (1988) Perception of the length of voluntary movements. Somatosens Res 5:335-348

Jaensch E (1906) Über Täuschungen des Tastsinns. Z Psychol 41:280-294

Lamb GD (1983) Tactile discrimination of textured surfaces: psychophysical performance measurements in humans. J Physiol 338:551-565

Lederman SJ (1974) Tactile roughness of grooved surfaces: the touching process and effects of macro- and microsurface structure. Percept Psychophys 16:385-395
Lederman SJ (1983) Tactual roughness perception: spatial and temporal determinants. Can J Psychol 37:498-511

Meftah EM, Belingard L, Chapman CE (2000) Relative effects of the spatial and temporal characteristics of scanned surfaces on human perception of tactile roughness using passive touch. Exp Brain Res 132:351-361

Nefs HT, Kappers AML, Koenderink JJ (2002) Frequency discrimination between and within line gratings by dynamic touch. Percept Psychophys 64:969-980

Otto Roelofs C, Zeeman WPC (1951) Influence of different sequences of optical stimuli on the estimation of duration of a given interval of time. Acta Psychol (Amst) 8:89-128

Parrish CS (1893) VIII. The cutaneous estimation of open and filled space. Am J Psychol 6:514-523

Révész G (1934) System der optischen und haptischen Raumtäuschungen. Z Psychol 131:295-375

Sanders AFJ, Kappers AML (2008) Curvature affects haptic length perception. Acta Psychol (Amst) 129(3):340-351

Smith AM, Chapman CE, Deslandes M, Langlais J-S, Thibodeau M-P (2002) Role of friction and tangential force variation in the subjective scaling of tactile roughness. Exp Brain Res 144:211-223

Suzuki K, Arashida R (1992) Geometrical haptic illusions revisited: Haptic illusions compared with visual illusions. Percept Psychophys 52:329-335

Thomas EAC, Brown I Jr (1974) Time perception and the filled-duration illusion. Percept Psychophys 16:449-458

Vega-Bermudez F, Johnson KO, Hsiao SS (1991) Human tactile pattern recognition: active versus passive touch, velocity effects, and patterns of confusion. J Neurophysiol 65:531-546

Von Skramlik E (1933) Über die Beeinflussung unserer Tastwahrnehmungen durch Richtung und Schnelligkeit der Tastbewegung. Z Sinnesphysiol 64:97-114

Wapner S, Weinberg J, Glick JA, Rand G (1967) Effect of speed of movement on tactual-kinesthetic perception of extent. Am J Psychol 80:608-613

Wong TS (1977) Dynamic properties of radial and tangential movements as determinants of the haptic horizontal-vertical illusion with an L figure. J Exp Psychol Hum Percept Perform 3:151-164 